\title{
A Cluster based Routing Protocol for MANET using Mahalanobis Distance based Clustering and Gravitational Search Algorithm
}

\author{
${ }^{1}$ T.V.Suresh Kumar, ${ }^{2}$ Dr.Prabhu G Benakop \\ ${ }^{1}$ Research Scholar, Electronics and Communication Engineering, JNTUH Hyderabad, Telangana, India. \\ tv.sureshkumar18@gmail.com \\ ${ }^{2}$ Principal, Methodist College of Engg \& Tech., Hyderabad, Telangana, India.
}

\section{ABSTRACT}

Mobile ad hoc network is generally an independent wireless network in the mobile nodes are communicated through the wireless links without using any centralized infrastructure. Since, the dynamic topology of the network affects the routing and data transmission between the nodes. In this research, an effective clustering and routing methods are developed to improve the MANET performances. The mahalanobis distance based clustering algorithm is used to select an effective cluster head from the network. Then gravitational search algorithm is used to find a routing path from the source to the destination over the cluster heads. The fitness function of gravitational search algorithm considers the multi objective such as residual energy, distance and node degree for optimizing the network performances. The performance of the proposed method are taken in terms of average packet delivery ratio, energy consumption, average end to end delay and normalized control overhead. The performance of the proposed method is compared to with two exiting works such as FEER and FHACO.The energy consumption of proposed method is 940J at 300 data packets that is less when compared to the FEER and FHACO.

Key words: Cluster head, gravitational search algorithm, mahalanobis distance based clustering, mobile ad hoc network, residual energy and routing path.

\section{INTRODUCTION}

Mobile Ad hoc Network (MANET) is a collection of selfsustaining mobile nodes that communicates with other nodes over the wireless connections. Since, the MANET is a one of the wireless ad hoc network that contains various properties such as self-healing, peer-to-peer and self forming network. This MANET doesn't requires any pre-existing infrastructure to perform the communication among the mobile nodes [1] [2]. The mobile nodes in the dynamic mobile network is act as router as well as host [3]. If the nodes are not in the transmission range of the source node, that requires other nodes for transmit the data to the respective destination [4]. The ad hoc networks are used in various situations such as rescue operations, emergency search, vehicular communications, military applications and conferences [5]. The instability and vulnerability are occurred in the node and link of MANET, due to the irregular mobility, huge difference of received signal strength and less battery power [6]. Additionally, the time varying topology and node's mobility causes higher overhead while handling the changes of routes [7].

The node's higher mobility in network environment creates the frequent link failure. This causes the heavy packet losses over the network. Moreover the network partition is created in the network, due to the nodes mobility. The network partition affects the packet transmitting rate [8]. The aforesaid limitations are overcome by using an effective clustering and routing techniques. The clustering technique is used to minimize the traffic in the distributed MANET. The Cluster Head $(\mathrm{CH})$ selection is an important phase of the clustering algorithm. Since, the $\mathrm{CH}$ selection is carried out by two methods connectivity based and identity-based algorithm [9]. The information about the local link connectivity is important consideration in the route establishment and maintenance of MANET [10]. The design of routing protocol which adopts the topology variation is important for supporting the Quality of Service (QoS). Because, the frequent link break of high-speed node environment affects the QoS performance [11]. For example, the existing protocols used in the MANET are specified as follows: binary particle swarm optimization algorithm [12], election algorithm [13], power aware heterogeneous AODV routing protocol [14] and loose-virtualclustering-based routing protocol [15]. The major contributions of this research paper are given as follows:

- A reliable data transmission is achieved by combining both the clustering and routing techniques in the 
T.V.Suresh Kumar et al., International Journal of Advanced Trends in Computer Science and Engineering, 9(4), July - August 2020, 4201 - 4208

MANET. Since, the clustering technique is used to minimize the traffic in the network.

- The Mahalanobis Distance Based Clustering (MDBC) algorithm is developed in the MANET to form the appropriate clusters and to select the optimal $\mathrm{CH}$ from the clusters. The main advantage of using MDBC in MANET is that considers the covariance of the node's position while calculating the distance.

- Additionally, the data transmission from the source to the destination over the $\mathrm{CHs}$ are detected by using the Gravitational Search Algorithm (GSA). Since, the GSA considers three fitness values such as residual energy, distance and node degree to overcome the node/link failure.

The overall organization of the paper is given as follows: the literature survey about the recent techniques related to the MANET is given in the section 2. The proposed clustering and routing algorithm using MDBC and GSA are clearly described in the section 3. The performance analysis of the proposed method is explained in the section 4. Finally the conclusion is made in section5.

\section{LITERATURE SURVEY}

The literature survey about the recent techniques related to the clustering and routing in MANET are explained in this section.

Pathak, S. and Jain, S [16] developed the Weight Based Clustering (WBC) protocol to perform the routing in MANET. This WBC considers two different parameters for clustering the network such as node degree and bandwidth requirement. The parameters considered in the WBC are used to derive the weight function and node's weight. Then the calculated weights are used in the cluster generation and $\mathrm{CH}$ selection. Moreover cluster merging is used in MANET for combining two clusters when the clusters are too near in the network. The stability of ensured by assigning the weight of the $\mathrm{CH}$. This work fails to analyze the energy consumption and delay during the data transmission.

Robinson, Y.H., Krishnan, R.S., Julie, E.G., Kumar, R. and Thong, P.H [17] presented the reliable routing method for obtaining the QoS in MANET. The node's signal strength from the deployed nodes are used to compute the bandwidth requirement. The delay and stability parameters helps to select the appropriate route over the network. The conventional broadcasting is incorporated in this reliable routing for identifying the effective route which accomplished by neighbor knowledge methods. The routing overhead is minimized by using the Neighbor Knowledge-based Rebroadcast (NKR) algorithm and Loose Virtual Clustering (LVC) algorithm. The performance of the designed algorithm is degraded and QoS is affected, due to the dynamic networks.

Rajashanthi, M. and Valarmathi, K [18] presented the effective energy-saving mechanism which is integrated with the MANET proactive routing protocol. An efficiency of the data transmission is identified by using the energy-aware routing method. Initially, the K-medoid clustering algorithm is used for generating the clusters. Since, this clustering minimizes the cost of data routing in large and dense networks. Subsequently, the Opposition Genetic-based Fish Swarm Optimization (OGFSO) algorithm is used to obtain the multipath routing for minimizing the energy consumption. This OGFSO causes higher delay during data transmission.

Rao, M. and Singh, N [19] developed the QoS based routing algorithm for transmitting the data over the MANET. Initially, the network is clustered by using the K-means clustering method. Subsequently, the firefly optimization is used for classifying the optimizing the grouped nodes to detect the $\mathrm{CHs}$ from its own clusters. Additionally, the data transmission between the nodes are carried out by using the TDMA based MAC routing method. The developed K-means with firefly and MAC routing is named as KF-MAC method. This KF-MAC method minimizes the packet loss rate. However, the delay is high, when the data flows under labelled packet exchanging protocols.

Kandan, J.M. and Sabari, A [20] developed the Fuzzy Hierarchy Ant Colony Optimization (FHACO) routing protocol for selecting the appropriate $\mathrm{CH}$. This selection of appropriate $\mathrm{CH}$ is helps to control the data packet transmission in the cluster. The weight of the nodes decides the normal nodes to become a $\mathrm{CH}$. The parameters used for calculating the weight of the nodes are node degree, buffer size, energy and speed. This FHACO increases the routing overhead and throughput over the network. The probability distribution of ACO is changed for each iteration.

\section{PROPOSED METHOD}

In this proposed method, the reliable data transmission is carried out by using an effective clustering and routing techniques. Initially, the MDBC is developed to group the nodes in the network as well as it selects an effective $\mathrm{CHs}$ from the respective clusters. The data from the normal nodes are collected by its $\mathrm{CH}$. Subsequently, the optimal path from the source to the destination is identified by using the GSA algorithm. The overall flowchart for the proposed method is shown in the Figure 1. 


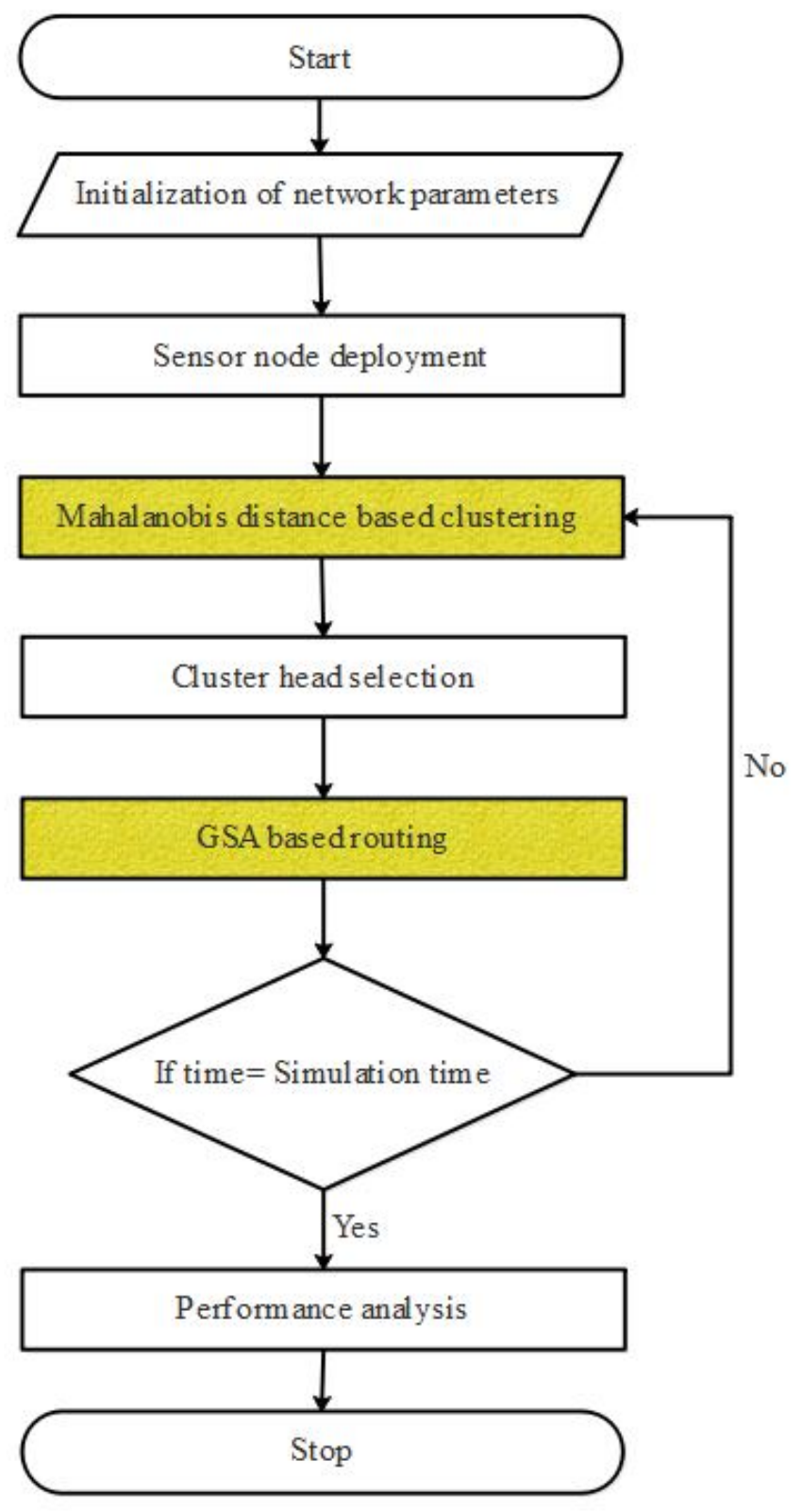

Figure 1: Flowchart of the proposed method

\subsection{Mahalanobis distance based clustering}

The MDBC is introduced in the proposed method for improving the network performance. Since, the different distance from the destination node causes unbalanced energy consumption over the network. The mahalanobis distance from the node to destination node is used to cluster the nodes in the MANET. Additionally, the appropriate $\mathrm{CHs}$ are identified by using this clustering. The threshold to select the candidate node as $\mathrm{CH}$ is expressed in the equation (1).

$$
T(n)=\frac{p}{1-p\left(\operatorname{rmod} \frac{i}{p}\right)}+(1-p) \frac{\max _{\text {Dist }}-M D_{i}}{\max _{\text {Dist }}-\min _{\text {Dist }}}\left(\frac{E_{R}}{E_{0}}\right)
$$

Where, $n$ is the random number among the 0 and $1 ; i$ is the number of nodes: probability of a candidate node become $\mathrm{CH}$ is represented as $p$; the number or round is denoted as $r$; The node's initial energy and residual energy are represented as $E_{0}$ and $E_{R}$ respectively. The maximum and minimum distance from the node to destination node are denoted as $\max _{D i s t}$ and min $_{\text {Dist }}$. Moreover, the $M D_{i}$ specifies the mahalanobis distance which is expressed in the equation (2).

$$
M D_{i}=\sqrt{(\vec{x}-\vec{y})^{T} S^{-1}(\vec{x}-\vec{y})}
$$

Where, the $x$ andyspecifies the location of the nodes. Then the attributes of $x$ and $y$ axis are represented as $\vec{x}$ and $\vec{y}$ respectively. Besides, $S^{-1}$ specifies the covariance among the $\vec{x}$ and $\vec{y}$. An unwanted information during the distance calculation is reduced by considering the correlation among the $\vec{x}$ and $\vec{y}$ in the mahalanobis distance. This helps to enhance the accuracy while calculating the accuracy.

This MDBC algorithm clusters the network as well as it selects an effective $\mathrm{CH}$ from its clusters. The location information about the identified $\mathrm{CHs}$ are given to the GSA based routing to identify the optimal routing path through the MANET.

\subsection{Gravitational search algorithm based routing}

GSA is generally a population search optimization algorithm which is motivated by the newton first law of gravity. In GSA, the particles are attracted to other particles based on the gravitational force that is directly proportional to product of masses and inversely proportional to the distance among the particles. The newton $2^{\text {nd }}$ law states that each particle acceleration is mainly depends on the applied force and particle's mass.

The swarm of agents considered in the GSA is $O_{B}$ and the each agent of GSA is $B_{j}, 1<j<O_{B}$ which specifies the complete solution. The position and velocity of an each agent are $Z_{j}^{d}$ and $V_{j}^{d}$, where $1<d<D$, the dimension of the search space is represented as $d$.

The $j$ th agent position, when there is a $O_{B}$ amount of agents in the GSA is represented as $Z_{J}=\left\{z_{j}^{1}, z_{j}^{2}, \ldots, z_{j}^{D}\right\}$, where $j$ th agent position in dimension $d$ is denoted as $z_{j}^{D}$. Equation (3) shows the force acted on the agent $j$ from $k$ th agent.

$$
F_{j k}^{d}(t)=G(t) \frac{M_{P j}(t) \times M_{a k}(t)}{E_{j k}(t)}\left(z_{k}^{d}(t)-z_{j}^{d}(t)\right)
$$

Where, active gravitational mass with respect to the $k$ th agent is represented as $M_{a k}(t)$; passive gravitational mass with 
T.V.Suresh Kumar et al., International Journal of Advanced Trends in Computer Science and Engineering, 9(4), July - August 2020, 4201 - 4208

respect to the $j$ th agent is represented as $M_{P j}(t)$; gravitational constant in time $t$ is represented as $G(t)$ and Euclidian distance among the agent $j$ and $k$ is $E_{j k}$. Moreover, the total amount of force used by the all agents on the agent $j$ at dimension $d$ in time $t$ is represented in equation (4).

$$
F_{j}^{d}(t)=\sum_{k=1, j \neq k}^{O_{B}} \operatorname{rand}_{k} \times F_{j k}^{d}(t)
$$

The gravitational and inertial masses for $B_{j}$ agent are determined by using the fitness calculation given in the equation (5). Here, the agent with less masses is considered as less capable than the remaining agents.

$$
m_{j}(t)=\frac{\text { fit }_{j}-\operatorname{worst}(t)}{\operatorname{Best}(t)-\operatorname{worst}(t)}
$$

Where, the $j$ th agent fitness function at time $t$ is represented as fit $_{j}(t)$. Additionally, Best $(t)$ and $\operatorname{worst}(t)$ are expressed in equation (6) and (7) respectively. Since, Best( $t)$ and $\operatorname{worst}(t)$ are defined for minimization problem.

$$
\begin{aligned}
\operatorname{Best}(t) & =\min \text { fit }_{j}(t) j \in\left\{1,2, \ldots, O_{B}\right\} \\
\operatorname{Worst}(t) & =\max \text { fit }_{j}(t) j \in\left\{1,2, \ldots, O_{B}\right\}
\end{aligned}
$$

GSA,

The following equation (8) shows the masses of the

$$
M_{a j}=M_{P j}=M_{j j}=M_{j}, \quad M_{j}=\frac{m_{j}(t)}{\sum_{k=1}^{O_{B}} m_{k}(t)}
$$

The $j$ th agent acceleration in direction $d$ at time $t$ is defined based on the motion law which is shown in the equation (9).

$$
a_{j}^{d}=\frac{F_{j}^{d}(t)}{M_{j}(t)}
$$

Therefore, the agents with higher masses have minimum fitness value which is considered as effective agents. The agents with higher masses attracts the remaining masses. Moreover, the forces from the higher mass and higher acceleration affects the agents with less masses. The optimal solution is discovered by updating the velocity and position of the agent. The velocity and position of the agent in the GSA are represented in the equation (10) and (11) respectively.

$$
\begin{array}{r}
V_{j}^{d}(t+1)=\operatorname{rand} \times V_{j}^{d}(t)+a_{j}^{d} \\
Z_{j}^{d}(t+1)=Z_{j}^{d}(t+1)+V_{j}^{d}(t+1)
\end{array}
$$

\subsubsection{Identification of optimal path for data transmission}

The $j$ th agent defines the complete solution in the GSA algorithm and the agent denotes the CH's optimal location obtained from the distance based clustering. Here, the dimension of all agent is identical to the amount of CHs. Consider $Z_{J}=\left\{z_{j}^{1}, z_{j}^{2}, \ldots, z_{j}^{N}\right\}$ which defines the agent $j$ in the population, $1<j<O_{B}$, where the amount of $\mathrm{CHs}$ in the network is $N$; amount of agents are represented as $O_{B}$; component in the $j$ th agent is $z_{j}^{d}, 1<d<N$ specifies the sensor's coordinates.

\subsubsection{Fitness function}

The fitness function used in the GSA for identifying the optimal path considers three different values such as residual energy of the $\mathrm{CH}$, distance and node degree.

\section{a. Residual energy}

The primary objective considered in the fitness function is the residual energy of the $\mathrm{CH}\left(E_{C H}\right)$. The node with higher energy has higher priority for transmitting/ receiving the data from the cluster members. The first objective $\left(c_{1}\right)$ is expressed in the equation (12).

$$
c_{1}=\sum_{j=1}^{N} E_{C H_{j}}
$$

\section{b. Distance}

The distance is considered as secondary objective $\left(c_{2}\right)$ during route generation, which is given in equation (13). Here, two kinds of differences are considered such as distance from the $\mathrm{CH}$ to the Next Hop $(\mathrm{NH})$ node $\left(\operatorname{dist}\left(\mathrm{CH}_{j}, \mathrm{NH}\right)\right.$ ) and distance from the $\mathrm{NH}$ to the destination node (dist $(\mathrm{NH}, \mathrm{DN}))$, where $D N$ represents the destination node.

$$
c_{2}=\frac{1}{\sum_{j=1}^{N} \operatorname{dist}\left(\mathrm{CH}_{j}, \mathrm{NH}\right)+\operatorname{dist}(\mathrm{NH}, \mathrm{DN})}
$$

\section{c. Node degree}

The node degree specifies the normal nodes present in the $\mathrm{NH}$ node. The amount of energy consumption while receiving the data is less, when the NH node's node degree is less in the network. The first objective $\left(c_{3}\right)$ is expressed in the equation (14).

$$
c_{3}=\frac{1}{\sum_{j=1}^{N} H_{j}}
$$

Where, the $H_{j}$ specifies the number of nodes in the respective $\mathrm{CH}$.

The computed objective values are conflicted each other, so weighted sum approach is used and a specific weight value is 
T.V.Suresh Kumar et al., International Journal of Advanced Trends in Computer Science and Engineering, 9(4), July - August 2020, 4201 - 4208

assigned to each objective value. Here the multiple objectives are converted into single objective which is shown in equation (15).

$$
f i t=\gamma_{1} \times c_{1}+\gamma_{2} \times c_{2}+\gamma_{3} \times c_{3}
$$

$$
\text { Where } \sum_{j=1}^{3} \gamma_{j}=1, \quad \gamma_{j} \in(0,1)
$$

Therefore, an optimal transmission path is identified by using the GSA algorithm. After identifying the transmission path, the data packets are transmitted from the source to the destination.

In this proposed method, the CH's residual energy is monitored at each simulation time to avoid the route failure through the network. The clustering algorithm is initiated again in the network, when any of the $\mathrm{CHs}$ energy level goes beyond the threshold level. Additionally, the faster convergence of the GSA is used to obtain an optimal routing path. The nodes with inadequate residual energy doesn't considered in the routing path. Because, the node failure during data transmission causes the packet loss. After determining the routing path, the data packets are transferred through the network.

\section{RESULTS AND DISCUSSION}

The experimental and comparative analysis of the proposed method is described in this section. The implementation and simulation of this MANET is carried out by using the Network Simulator-2.35. In MANET, the MDBC is developed for identifying the CHs through the network. Subsequently, the GSA based algorithm is developed for creating the transmission path from the source to the destination node. Here, 100 nodes are considered to be deployed over the area of $1500 \mathrm{~m} \times 1500 \mathrm{~m}$. The specification parameters considered in this proposed method is specified in the Table 1.

Table 1: Specification parameters

\begin{tabular}{|l|l|}
\hline Parameters & Value \\
\hline Area & $1500 \mathrm{~m} \times 1500 \mathrm{~m}$ \\
\hline Clustering algorithm & MDBC \\
\hline Routing algorithm & GSA \\
\hline Number of nodes & 100 \\
\hline Number of packets & $20-300$ \\
\hline Mobility model & Random way point model \\
\hline Speed of node & $0-25 \mathrm{~ms}$ \\
\hline Transmission range & $250 \mathrm{~m}$ \\
\hline Simulation time & $50-500 \mathrm{~s}$ \\
\hline
\end{tabular}

\subsection{Performance analysis}

The performance analysis of the proposed method is taken in terms of average Packet Delivery Ratio (PDR), energy consumption, average End to End Delay (EED) and normalized routing control overhead. Additionally, these performances are compared with FEER [20] and FHACO [20]. This FEER [20] and FHACO [20] are also implemented and simulated for the specifications mentioned in the Table 1 . The performances are taken by varying the different number of data packets.

\subsubsection{Packet delivery ratio}

PDR is ratio between the amount of packets received in the destination and amount of delivered packets by the source node. Since, the PDR is mainly depends on the packets which is transmitted and collected while delivering the packets.

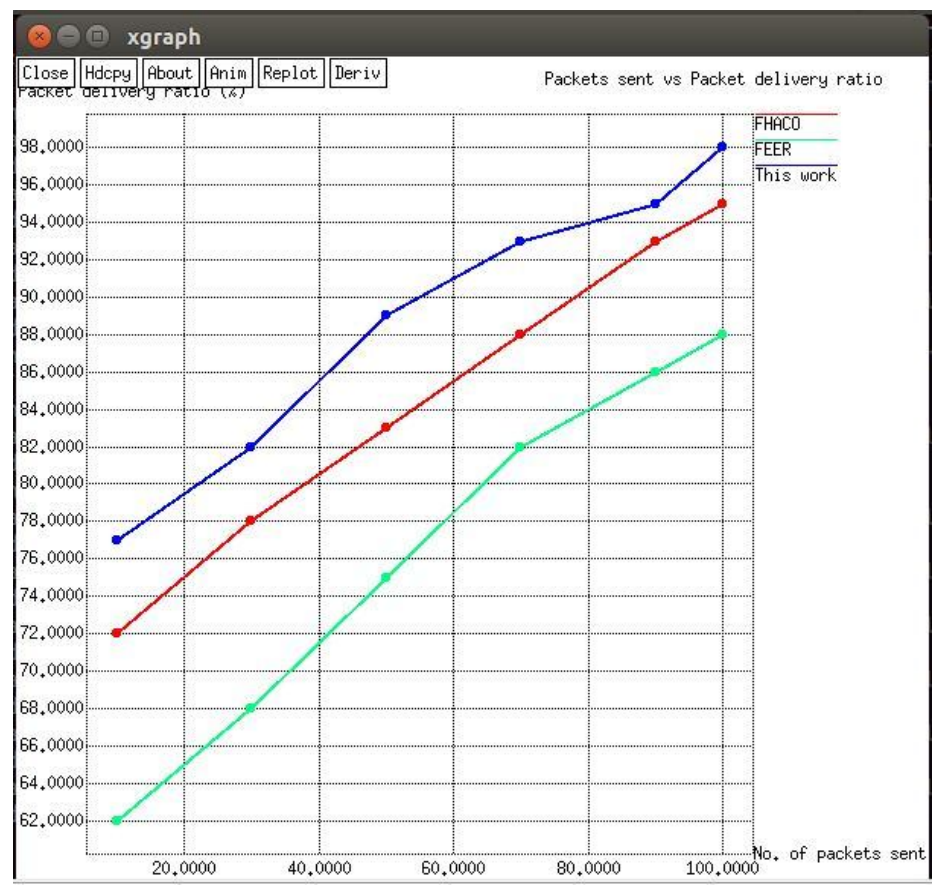

Figure 2: Packet delivery ratio

Figure 2 shows the comparative analysis of PDR for proposed method with FEER [20] and FHACO [20]. This PDR comparison is made by varying the amount of packets delivered through the network. From the Figure 2, concludes that the proposed method higher PDR than the existing works. For example, the PDR of the proposed method is $98 \%$ at 100data packets which is high when compared to the existing works. The proposed method achieves the higher PDR by considering the residual energy of the nodes in the fitness function of GSA. The residual energy consideration is used to avoid the node with lesser energy during the data transmission. Because the node with less residual energy causes the node failure which causes the packet loss through the network. 
T.V.Suresh Kumar et al., International Journal of Advanced Trends in Computer Science and Engineering, 9(4), July - August 2020, 4201 - 4208

\subsubsection{Energy consumption}

The calculation of energy consumption is depends on the amount of energy consumed by the energy during the packet routing in MANET. The energy consumption is the produce of the sensor nodes, power and time. Moreover, the power and time is considered in terms of watts and seconds respectively.

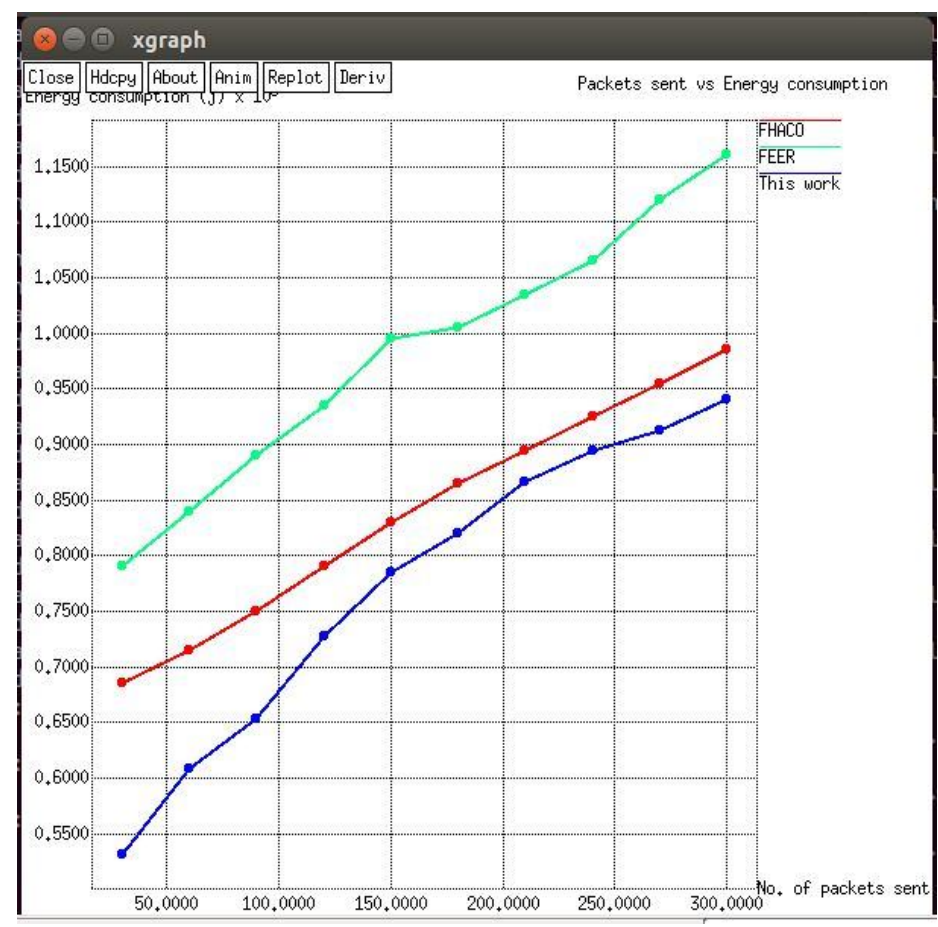

Figure 3: Energy consumption

The energy consumption of the proposed method with FEER [20] and FHACO [20] is shown in the Figure 3. The comparison shown in the Figure 3 is taken by varying the number of packets from 30 to 300 . From the Figure 3, knows that the energy consumption of the proposed method is less when compared to the FEER [20] and FHACO [20]. For the instance, the energy consumption of the proposed method is $940 \mathrm{~J}$ at 300 packets, which is less when compared to the existing works. The MDBC used in the proposed method precisely identifies the $\mathrm{CH}$ over the MANET. This helps to reduce the unwanted energy consumption. Moreover, the distance consideration in the GSA is used to detect the shortest path over the MANET. The energy consumption of the network is minimized by identifying the shortest path over the network.

\subsubsection{Average end to end delay}

EED is defined as the amount of time taken for transmitting and receiving the data packets over the network.

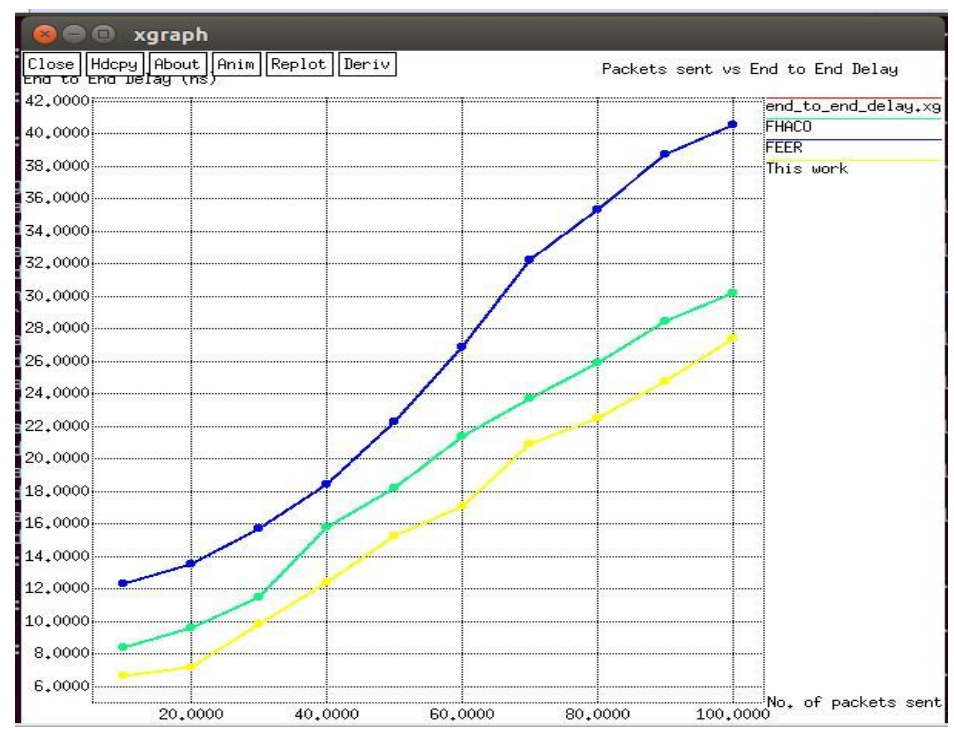

Figure 4: Average end to end delay

Figure 4 shows the comparative analysis of average EED for proposed method with FEER [20] and FHACO [20]. This average EED comparison is made by varying the amount of packets from 10 to 100. From the Figure 4, knows that the proposed method less EED than the existing works. For example, the average EED of the proposed method is $27.4 \mathrm{~ns}$ at 100 data packets that is less when compared to the existing works. The lesser delay over the MANET is achieved by considering the distance factor in both the clustering and routing while routing the data to the destination.

\subsubsection{Normalized control overhead}

Normalized Control overhead is defined as total amount of unaffected packets are provided while transmitting the data packets.

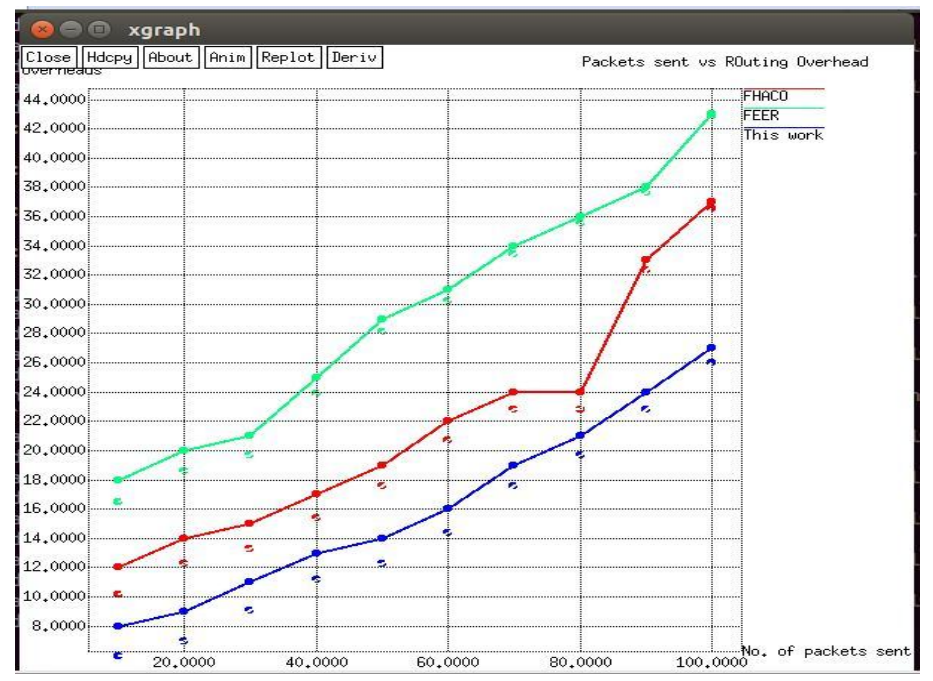

Figure 5: Normalized control overhead 
The normalized control overhead of the proposed method with FEER [20] and FHACO [20] is shown in the Figure 5. The comparison shown in the Figure 5 is taken by varying the number of packets from 10 to 100 . From the Figure 5, knows that the normalized control overhead of the proposed method is less when compared to the FEER [20] and FHACO [20]. For example, the overhead of the proposed method is 27 at 100 data packets that is less than the existing works. The fitness function values of GSA such as residual energy, distance and node degree are used to minimize the losses while transmitting the data packets. This helps to minimize the routing overhead in the MANET.

\section{CONCLUSION}

In this paper, the MDBC algorithm and GSA routing is combined for achieving the better data transmission through the network. The MDBC algorithm used in this proposed method considers the covariance during distance calculation which helps to select the significant $\mathrm{CH}$. Additionally, the faster convergence of the GSA selects an optimal path from the source to the destination. The GSA used in the proposed method considers three values such as residual energy, distance and node degree. Therefore the developed clustering and routing overcome the issues of link/ node failure caused by the dynamic network topology and less battery power. The proposed method shows better performances than the FEER and FHACO. The control overhead is 27 at 100 data packets that is less than the FEER and FHACO methods.

\section{REFERENCES}

1. Selvi, P.T. and GhanaDhas, C.S., 2019. A novel algorithm for enhancement of energy efficient zone based routing protocol for MANET. Mobile Networks and Applications, 24(2), pp.307-317.

https://doi.org/10.1007/s11036-018-1043-X

2. Singh, G., Kumar, N. and Verma, A.K., 2014. Antalg: An innovative aco based routing algorithm for manets. Journal of Network and Computer Applications, 45, pp.151-167.

3. Malathi, M. and Jayashri, S., 2016. Modified Bi-directional Routing with Best Afford Path (MBRBAP) for Routing Optimization in MANET. Wireless Personal Communications, 90(2), pp.861-873.

https://doi.org/10.1007/s11277-016-3233-9

4. Wang, Z., Chen, Y. and Li, C., 2013. PSR: A lightweight proactive source routing protocol for mobile ad hoc networks. IEEE transactions on Vehicular Technology, 63(2), pp.859-868.

5. Santhi, G. and Nachiappan, A., 2012. Fuzzy-cost based multiconstrained QoS routing with mobility prediction in MANETs. Egyptian informatics journal, 13(1), pp.19-25.

https://doi.org/10.1016/j.eij.2011.12.001
6.Biradar, R.C. and Manvi, S.S., 2012. Neighbor supported reliable multipath multicast routing in MANETs. Journal of Network and Computer Applications, 35(3), pp.1074-1085.

7. Zhang, Q.J., Wu, M.Q., Yan, Z.H.E.N. and Shang, C.L., 2010. AODV routing overhead analysis based on link failure probability in MANET. The Journal of China Universities of Posts and Telecommunications, 17(5), pp.109115.

8. Gopinath, S. and Nagarajan, N., 2015. Energy based reliable multicast routing protocol for packet forwarding in MANET. Journal of applied research and technology, 13(3), pp.374-381.

9. Ramesh, S. and Smys, S., 2017. A software-based heuristic clustered (sbhc) architecture for the performance improvement in manet. Wireless Personal Communications, 97(4), pp.6343-6355.

10. Han, S.Y. and Lee, D., 2013. An adaptive hello messaging scheme for neighbor discovery in on-demand MANET routing protocols. IEEE communications letters, 17(5), pp.1040-1043.

11. Chen, Z., Zhou, W., Wu, S. and Cheng, L., 2020. An Adaptive on-Demand Multipath Routing Protocol WithQoS Support for High-Speed MANET. IEEE Access, 8, pp.44760-44773.

https://doi.org/10.1109/ACCESS.2020.2978582

12.Jamali, S., Rezaei, L. and Gudakahriz, S.J., 2013. An energy-efficient routing protocol for MANETs: a particle swarm optimization approach. Journal of applied research and technology, 11(6), pp.803-812.

13. Singh, S., Rajpal, N. and Sharma, A., 2014. Address allocation for MANET merge and partition using cluster based routing. SpringerPlus, 3(1), p.605.

14. Safa, H., Karam, M. and Moussa, B., 2014. PHAODV: Power aware heterogeneous routing protocol for MANETs. Journal of Network and Computer Applications, 46, pp.60-71.

15. Zhao, P., Yang, X., Yu, W. and Fu, X., 2013. A loosevirtual-clustering-based routing for power heterogeneous MANETs. IEEE Transactions on vehicular technology, 62(5), pp.2290-2302.

https://doi.org/10.1109/TVT.2012.2237556

16. Pathak, S. and Jain, S., 2016. A novel weight based clustering algorithm for routing in MANET. Wireless Networks, 22(8), pp.2695-2704.

17. Robinson, Y.H., Krishnan, R.S., Julie, E.G., Kumar, R. and Thong, P.H., 2019. Neighbor Knowledge-based Rebroadcast algorithm for minimizing the routing overhead in Mobile Ad-hoc Networks. Ad Hoc Networks, 93, p.101896.

18. Rajashanthi, M. and Valarmathi, K., 2020. Energyefficient multipath routing in networking aid of clustering with OGFSO algorithm. Soft Computing, pp.1-10.

19. Rao, M. and Singh, N., 2018. Energy efficient QoS aware hierarchical KF-MAC routing protocol in MANET. Wireless Personal Communications, 101(2), pp.635-648.

20.Kandan, J.M. and Sabari, A., 2019. Fuzzy hierarchical ant colony optimization routing for weighted cluster in MANET. Cluster Computing, 22(4), pp.9637-9649. https://doi.org/10.1007/s10586-017-1318-1

21. Dr. Ramesh.Vatambeti, N. B. D. B. V. (2020). Optimal

Routing and Load Balancing based Congestion Avoidance 
in MANET using Improved Ad-Hoc On-Demand Distance Vector Routing. International Journal of Control and Automation, 13(02), 110 - 127.

22. Ramesh. Vatambeti, D.Pramodh Krishna, K.SangeethaSupriya, (2020) "A Novel Scheme for Energy Conservation and reduction in Routing Overhead of AODV for Wireless Ad-Hoc Networks", International Journal of Advanced Science and Technology, 29(3), 5281 - 5287. Retrieved from

http://sersc.org/journals/index.php/IJAST/article/view/6034.

23. B.Nanditha, Dr V Ramesh, B.Veeramallu, "Achieving Energy Efficiency and Increasing the Network Life Time in MANET through Fault Tolerant Multi-Path Routing', International Journal of Intelligent Engineering and Systems, Vol.10, No.3, 2017 DOI: 10.22266/ijies2017.0630.18.

24. Pooja Singh, Nasib Singh Gill, 2019. A Secure and Power-Aware Protocol for Wireless Ad Hoc Networks, International Journal of Advanced Trends in Computer Science and Engineering, 8(1), PP. 34-41

https://doi.org/10.30534/ijatcse/2019/07812019

25. G.T. Chavan, VemuruSrikanth, 2019. Effective Zone Based Routing Protocols forMANET, International Journal of Advanced Trends in Computer Science and Engineering, 8(5), PP. 34-41

https://doi.org/10.30534/ijatcse/2019/26852019 